\title{
The rank of the automorphism group of a finite group
}

\author{
B. A. F. Wehrfritz ${ }^{1}$
}

Received: 5 April 2018 / Published online: 9 July 2018

(C) The Author(s) 2018

\section{Abstract}

Let $G$ be a finite group whose order is divisible by $e$ primes (counting repetitions). Then the automorphism group of $G$ has Prüfer rank at most $\mathrm{e}^{2}$, meaning that each subgroup of the automorphism group of $G$ can be generated by at most $\mathrm{e}^{2}$ elements.

Keywords Finite group · Automorphism group · Prüfer rank

Mathematics Subject Classification 20D45

\section{Introduction}

If $G$ is a finite group, then $d(G)$ denotes its minimal number of generators, $e(G)$ the number of prime divisors (counting repetitions) of its order $|G|$ and $r k(G)$ its rank; that is, the maximum of the $d(X)$ as $X$ ranges over the subgroups of $G$. Clearly $d(G) \leq r k(G) \leq e(G)$. Also [r] denotes the largest integer not exceeding the real number $r$.

Theorem 1 If $G$ is a finite group, then always $r k($ Aut $(G)) \leq e(G)+\left[e(G)^{2} / 2\right] \leq$ $e(G)^{2}$.

I mention and use $r k(\operatorname{Aut}(G)) \leq e(G)^{2}$ in [12] as if it is well-known. It seems this is not the case and I have failed to find any proof or indeed any mention of it in the literature. Thus I feel now the need to publish a proof. My proof is in the main elementary. The only really non-elementary facts I use are that if $S$ is a finite perfect simple group, then $d(S)=2, \operatorname{rk}(\operatorname{Out}(S)) \leq 5,|S|$ is divisible by 4 and $e(S) \geq 4$ (even 5 unless $S=A l t(5)$ ). I also use results from [5,8], see below for details, although these can be avoided at the expense of substantially lengthening the proof by using Theorem 2 below to reduce to the soluble case.

\section{Communicated by F. de Giovanni.}

$凶$ B. A. F. Wehrfritz

b.a.f.wehrfritz@qmul.ac.uk

1 Queen Mary University of London, London E1 4NS, England, UK 
Clearly $r k(G) \leq e(G)$ and it is easy to prove that $|A u t(G)| \leq|G|^{d(G)} \leq|G|^{e(G)}$, but these easy facts seem to be of no help in proving the theorem. Note also that $r k(A u t(G))$ need not be bounded by $(r k(G))^{2}$, though in significant special cases (e.g. $G$ elementary abelian) it is. As an obvious counter example, the cyclic group $G$ of order 8 has $r k(G)=1$ and $\operatorname{rk}(\operatorname{Aut}(G))=2$. More generally it is easy to see that if $G$ is the direct product of $r$ cyclic groups of order $2^{f}$ for $f \geq 3$ and $r \geq 1$, then $\operatorname{Aut}(G)$ contains an elementary abelian subgroup of rank $r^{2}+r$. Hence here $r k(G)=r$ while $r k($ Aut $(G)) \geq r^{2}+r>r^{2}$. (Also $e(G)^{2} / 2=(f r)^{2} / 2>r^{2}+r$ of course.)

Our second theorem below we originally used in the proof of Theorem 1 essentially to reduce to the soluble case, but thanks to $[5,8]$, which only very recently were brought to my attention, we no longer need to do this (At the end of this paper we sketch this alternative approach to Theorem 1). However, I think Theorem 2 is of independent interest.

Theorem 2 Let $G$ be a finite subgroup of $G L(n, F)$, where $n$ is a positive integer and $F$ is a field of positive characteristic $p$. Suppose $G$ has a soluble normal subgroup $S$ such that $G / S$ is a direct product of $r$ perfect simple groups. Then $r \leq[n / 2]$.

Clearly this bound is attained for all $n$ and all $F$ with $|F|>3$; recall $G L(2,2)$ and $G L(2,3)$ are soluble.

\section{Proof of the results}

By a theorem of Guralnick and Lucchini (independently), see [5,8], the rank of a finite group is bounded by one more than the maximum of the ranks of its Sylow subgroups. The following lemma is the analogue of Theorems 1 and 2 for the symmetric groups.

Lemma 1 For each positive integer $n$ we have $\operatorname{rk}(\operatorname{Sym}(n)) \leq 1+[n / 2]$. Further $\operatorname{rk}(\operatorname{Sym}(n))$ is 0 if $n=1$ and 1 if $n=2$. If $G$ is a subgroup of $\operatorname{Sym}(n)$ with a soluble normal subgroup $S$ such that $G / S$ is the direct product of s perfect simple groups, then $s \leq[n / 4]$.

Proof The rank of a Sylow $p$-subgroup of $\operatorname{Sym}(n)$ is $[n / p]$ for any prime $p$, see the Proposition of [10]. Thus the first claim follows from this and Guralnick and Lucchini's theorem. Clearly $G / S$ involves an elementary abelian 2-group of rank $2 s$ and by the Proposition of [10] again a Sylow 2-subgroup of $\operatorname{Sym}(n)$ has rank [n/2]. Therefore $s \leq[n / 4]$ (A very simple proof by induction on $n$ shows that at least $r k(\operatorname{Sym}(n)) \leq\left[n^{2} / 4\right]$, which would actually suffice for our purposes).

Note that Guralnick and Lucchini's theorem together with bounds in [10] yield that in Theorem 2 we have $r \leq 3 n / 4$ if $p>2$ and $r \leq 2 n$ if $p=2$. These are weaker than the claims of Theorem 2; also they do not seem to help with its proof.

Proof of Theorem 2 If $n=1$ we have $r=0$. Suppose $n=2$. If $G$ is metabelian then $r=0$. If not then $G$ is absolutely irreducible and hence is isomorphic to a subgroup of $G L\left(2, p^{f}\right)$ for some $f$ (e.g. [2] 29.21). Thus from Dickson's list of the subgroups 
of $\operatorname{PSL}\left(2, p^{f}\right)$, see [6] II.8.27, we have $r \leq 1$. Let $G$ be a counter example to the theorem with $n$ minimal. Then $n \geq 3$ and $r \geq 2$. Hence $G=G_{1} G_{2} \ldots G_{r}$, where the $G_{i}$ are normal subgroups of $G$, the $G_{i} / S_{i}$ are perfect simple for $S_{i}=G_{i} \cap S$ and $G / S$ is the direct product of the $G_{i} S / S$. We break the proof into a number of sublemmas.

(a) If $N$ is a normal subgroup of $G$, then $G / N$ and $N$ have the same structure as $G$. Specifically $N S / N$ is soluble and $G / N S$ is the direct product of the $G_{i} N S / N S$ (each being isomorphic to $G_{i} S / S$ or $\langle 1\rangle$ ). Also $N \cap S$ is soluble, $N /(N \cap S) \simeq$ $N S / S$ and the latter is the direct product of the $\left(N S \cap G_{i} S\right) / S$ (again each of which is isomorphic to $G_{i} S / S$ or $\langle 1\rangle$ ).

(b) We may assume that $\left(G_{i}\right)^{\prime}=G_{i}$ for each $i$.

Now $G_{i}$ is soluble-by-simple. Let $H_{i}$ be the soluble residual of $G_{i}$, so $\left(H_{i}\right)^{\prime}=H_{i}$ and $G_{i}=H_{i} S_{i}$. Also $T_{i}=H_{i} \cap S$ is the soluble radical of $H_{i}$ and $H_{i} / T_{i} \simeq G_{i} / S_{i}$. Thus if $H=H_{1} H_{2} \ldots H_{t}$ and $T=H \cap S$, then

$$
H / T=H_{1} T / T \times \cdots \times H_{r} T / T \simeq H_{1} / T_{1} \times \cdots \times H_{r} / T_{r} .
$$

Thus we may replace $G$ by $H$. Equivalently we may assume that $\left(G_{i}\right)^{\prime}=G_{i}$ for each $i$.

(c) We may assume $F$ is algebraically closed. Then $G$ is absolutely irreducible.

The first claim is obvious. Let $V=F^{(n)}$ taken as a right $G L(n, F)$-module in the obvious way and let $W$ be an $F G$-submodule of $V$ with $\{0\}<W<V$. If $G$ is a subgroup of $C_{G}(W) S \cap C_{G}(V / W) S$, then $G$ acts as a soluble group on both $W$ and $V / W$ and hence acts as a soluble group on $V$. But $G_{i}$ is not soluble. Thus each $G_{i} / S_{i}$ is avoided by at least one of $C_{G}(W)$ and $C_{G}(V / W)$ and then by the minimal choice of $n$ we have

$$
r \leq\left(\operatorname{dim}_{F} W\right) / 2+\left(\operatorname{dim}_{F}(V / W)\right) / 2=n / 2
$$

This contradiction shows that $G$ is irreducible.

(d) $G$ is primitive.

Suppose $V=V_{1} \oplus \cdots \oplus V_{t}$ is a system of imprimitivity of $G$ with $t \geq 2$. Set $N=\bigcap_{j} N_{G}\left(V_{j}\right)$. Then $G$ permutes the $V_{i}$ transitively and $G / N$ embeds into $\operatorname{Sym}(t)$. Hence $G / N$ involves at most $[t / 4]$ of the $G_{i} / S_{i}$ by Lemma 1 and thus $N$ must involve the remaining $G_{i} / S_{i}$, of which there are say $r_{1} \geq r-[t / 4]$. Let $I$ denote the set of all such $i$. Then $N /(N \cap S)$ is the direct product over $I$ of the $\left(G_{i} S \cap N\right) /(N \cap S)$. For each $i$ in $I$ the group $G_{i} S \cap N$ cannot act as a soluble group on all the $V_{\mathrm{i}}$ and hence there exists $j$ with $G_{i} S \cap N$ not contained in $C_{N}\left(V_{\mathrm{i}}\right)(N \cap S)$. Now the $G_{i}$ are normal in $G$ and $G$ permutes the $V_{i}$ transitively. Thus $G_{i} S \cap N$ is not contained in $C_{N}\left(V_{1}\right)(N \cap S)$ for each $i$ in $I$ and therefore $r_{1}=|I| \leq\left(\operatorname{dim}_{F}\left(V_{1}\right)\right) / 2$. Now $t \geq 2$ and $n=t \cdot \operatorname{dim}_{F} V_{1}$, so

$$
r \leq r_{1}+[t / 4] \leq\left(\operatorname{dim}_{F} V_{1}\right) / 2+t / 4 \leq n / 2 .
$$

This final contradiction yields that $G$ is primitive. 
(e) $E(G)$, the subgroup of $G$ generated by the subnormal quasi-simple subgroups of $\mathrm{G}$ is non-trivial.

For suppose $E(G)=\langle 1\rangle$. Set $N=\operatorname{Fitt}(G)$, the Fitting subgroup of $G$. Now every abelian normal subgroup of $G$ lies in $Z=\mathrm{F}^{*} 1_{n} \cap G$ by c), d) and [9] 1.13. (F* denotes the multiplicative group of $F$.) Also $N \geq Z=C_{G}(N)$ (e.g. see [11] 6.2 or [1] 31.13). Suppose $N$ is nilpotent of class $c \geq 3$. Then, with $\left\{\gamma^{i}(N)\right\}$ denoting the lower central series of $N,\left(\gamma^{c-1}(N)\right)^{\prime} \leq \gamma^{2 c-2}(N)=\langle 1\rangle$, so $\gamma^{c-1}(N) \leq Z$ and $N$ is not nilpotent of class $C$.

Consequently $c \leq 2$. In fact $c=2$, since if $N$ is abelian, then $N=Z=C_{G}(N)=$ $G$ and $r=0 \leq[n / 2]$. If $q$ is some prime and if $x$ and $y$ are elements of $N$ of order $q^{2}$ modulo $Z$, then $\left[x^{q}, y^{q}\right]=\left[x, y^{q \cdot q}\right]=1$. Hence $\left\langle x^{q}: x \in N,|x Z|=q^{2}\right\rangle$ is abelian and therefore lies in $Z$. Consequently every Sylow subgroup of $N / Z$ is elementary abelian.

Since $G$ is primitive, $N$ is homogeneous ([9] 1.7). Therefore $(N: Z)=\left(n^{\prime}\right)^{2}$ for some divisor $n^{\prime}$ of $n$, see [9] 3.2. Suppose $n^{\prime}=p_{1}^{e(1)} p_{2}^{e(2)} \ldots p_{t}^{e(t)}$, where the $p_{i}$ are distinct primes. Set $K=C_{G}(N / Z)$. Then $K^{\prime} \leq C_{G}(N)=Z$ and so $K$ is soluble. Also $G / K$ embeds into the direct product of the $t$-groups $G L\left(2 e(i), p_{i}\right)$. Now $G L(2,2)$ is soluble and $G L(4,2) \simeq \operatorname{Alt}(8)$. Neither involves the direct product of 2 perfect simple groups $(|\operatorname{Alt}(8)|=2.60 .168$ and is not divisible by $5^{2}$ ) and trivially $1 \leq[n / 2]$. Then $n^{\prime} \neq 2$ or 4 and so $\sum_{i} 2 . e(i)<n$ and $r \leq \sum_{i} e(i)<n / 2$. This final contradiction yields that $E(G) \neq\langle 1\rangle$.

(f) $E(G) . S \neq G$.

If $E(G) . S=G$, then $E(G)=E_{1} E_{2} \ldots E_{t}=E$ say, where the $E_{i}$ are the subnormal quasi-simple subgroups of $G$. Thus we can work with $E$ instead of $G$. If $r=0$ trivially $r \leq$ [n/2]. If $r=1$ clearly $n \geq 2$ and again $r \leq[n / 2]$. Thus $r \geq 2$. If $E$ is reducible, then $r \leq[n / 2]$, cf. c) above. Therefore $E$ is irreducible. Set $E_{0}=E_{1} E_{2} \ldots E_{r-1}$ and consider an irreducible $F E_{0}$-submodule $U$ of $V$. Now $\left[E_{0}, E_{r}\right]=\langle 1\rangle$, see $[1,11]$ again, so $U x \simeq U$ as $F E_{0}$-module for all $x \in E_{r}$ and clearly $U E_{r}=U E=V$. Thus $E_{0}$ is homogeneous and therefore $E_{0}$ acts faithfully on $U$.

Suppose $\operatorname{dim}_{F}(V / U) \geq 2$. By the minimal choice of $n$ we have $r-1 \leq(n-2) / 2$ and hence $r \leq[n / 2]$. Consequently $\operatorname{dim}_{F}(V / U) \leq 1$. If $\operatorname{dim}_{F}(V / U)=1$, then since $E_{0}$ is homogeneous and $U$ is irreducible we have $\operatorname{dim}_{F} U=1$. But then $E_{0}$ is diagonalizable and so abelian, which is false since $r>1$. Hence $U=V$. But then $E_{r} \leq C_{E}\left(E_{0}\right) \leq F^{*} 1_{n}$ (Schur's Lemma) and yet $E_{r}$ is not abelian. This final contradiction completes the proof of $\mathrm{f}$ ).

(g) The completion of the proof of Theorem 2.

Again set $E=E(G)$ and define $K \leq G$ by $S \leq K$ and $G / S=K / S \times E S / S$. By (e) and (f) we have $S<K<G$. Also [K,E] $E \cap S \leq Z=F^{*} 1_{n} \cap G$ by (d). Then $K$ stabilizes the series $\langle 1\rangle \leq Z \leq E Z$, so if $C=C_{\mathrm{G}}(E)$, then $K^{\prime} \leq C$. Hence $G=K E W=K^{\prime} E S=C E S$ and $C /(C \cap S)$ involves $G / E S$.

Neither $E$ nor $C$ is abelian. Thus if $U$ is an irreducible $F E$-submodule of $V$, then $V$ is a direct sum of copies of $U$ by (d), so $1<m=\operatorname{dim}_{F}(U)<n$ and $n=m k$ for some integer $k<n$. Further, by (c), (d) and [9] 1.15 the group $G$ is isomorphic to a subgroup of $(G L(m, F) \times G L(k, F)) / A$, where $A$ is a central subgroup of this direct 
product. Since $m<n$ and $k<n$, by the choice of $n$ we have $r \leq m / 2+k / 2 \leq n / 2$. This final contradiction completes the proof of Theorem 2.

If $S_{q}$ is a Sylow $q$-subgroup $G L(n, p), p$ prime, for some prime $q$, then $r k S$, is at most $n$ if $2<q \neq p$, is at most [3n/2] if $q=2 \neq p$ and is at most (less than if $n>2$ ) $n(n-1) / 2$ if $q=p$, see [10]. We define the integer function $s(n, p)$ of the positive integer variables $n$ and $p, p$ prime, as follows. Set $s(1, p)=1$ and $s(2,2)=2$. If $p>2$ set $s(2, p)=4, s(3, p)=5$ and $s(4, p)=7$. For all other values of $n$ and $p$ set $s(n, p)=n(n-1) / 2$. Notice that $s(n, p) \leq n+\left[n^{2} / 2\right] \leq n^{2}$ for all $n$ and $p$ and $s(n, p)<\left[n^{2} / 2\right]$ if $n>3$ or if $p=2<n$.

Lemma 2 We have $r k(G L(n, p)) \leq s(n, p)$ for all $n \geq 1$ and primes $p$.

Proof Clearly $r k(G L(1, p))=1$ and $r k(G L(2,2))=r k(\operatorname{Sym}(3))=2$, while $r k(G L(2, p)) \leq 4$; just check Dickson's list of the subgroups of $\operatorname{PSL}(2, p)$, see [6] II.8.27. The theorem from [5,8] together with the results from [10] quoted above yield the lemma.

It is easy to see that always $r k(G L(n, p)) \geq\left[n^{2} / 4\right]$ and that $r k(G L(n, p)) \geq$ [3n/2] if $p>2$. Lemma 2 is effectively the special case of the theorem where $G$ is elementary abelian.

Proof of Theorem 1 Set $e=e(G)$. We induct on $e$. If $e \leq 1$ the claim is clear, so assume $e \geq 2$. We have to prove that $r k(\operatorname{Aut}(G)) \leq e+\left[e^{2} / 2\right]$. Suppose $N$ is a characteristic subgroup of $G$ with $\langle 1\rangle<N<G$. There is an obvious homomorphism of $\operatorname{Aut}(G)$ into the direct product of $\operatorname{Aut}(N)$ and $\operatorname{Aut}(G / N)$ with say kernel $K$, where by stability theory $K$ is isomorphic to a section of $N^{e(G / N)}$, e.g. see [7] 1.C.3. Then $e(K) \leq e(N) \cdot e(G / N)$ and $e=e(N)+e(G / N)$. Induction on $e$ now yields that

$$
\begin{aligned}
r k(\operatorname{Aut}(G)) \leq & e(N)+\left[e(N)^{2} / 2\right]+e(G / N)+\left[e(G / N)^{2} / 2\right] \\
& +e(N) \cdot e(G / N) \leq \\
e+(e(N)+e(G / N))^{2} / 2= & e+e^{2} / 2 .
\end{aligned}
$$

But $r k(\operatorname{Aut}(G))$ is an integer, so $r k(\operatorname{Aut}(G)) \leq e+e\left[e^{2} / 2\right]$.

If no such $N$ exists then either $G$ is elementary abelian of rank $e$, when $r k(A u t(G)) \leq e+\left[e^{2} / 2\right]$ by Lemma 2 , or $G$ is a direct sum of say $t$ copies of a perfect simple group $S$. Assume the latter case. Now $d(S)=2, \operatorname{rk}(\operatorname{Out}(S)) \leq 5$, $r k(S) \leq e(S)$ and $e(S) \geq 4$. Then $r k(A u t(S)) \leq e(S)+5<e(S)+\left[e(S)^{2} / 2\right]$. This settes the case $t=1$.

Assume $t \geq 2$. Then $\operatorname{Aut}(G)$ is isomorphic to the wreath product of $\operatorname{Aut}(S)$ by $\operatorname{Sym}(t)$, where $\operatorname{Aut}(G)$ permutes transitively the copies of $S$ in the given direct decomposition of $G$. Therefore Lemma 1 yields that $r k(A u t(G)) \leq(e(S)+5) t+$ $1+[t / 2]$. Now $e=e(S) t, e(S) \geq 4, t \geq 2$ and $e \geq 8$. Hence

$$
r k(A u t(G)) \leq e+5 e / 4+e / 8+e / 8 \leq 5 e / 2 \leq 5 e^{2} / 16
$$

The theorem follows. 


\section{Final remarks}

We conclude with a sketch of a proof of Lemma 2 and hence of Theorem 1 that uses Theorem 2 but avoids using [5,8]. Let $G$ be a subgroup of $G L(n, p)$. As in the first sentence of the proof of Lemma 2 above we may assume $n \geq 3$. By results of Roggenkamp, see [3] 7.8, 7.9(i) and 7.20, there exists a Sylow subgroup $S$ of $G$ with $d(G) \leq \operatorname{pr}(G)+1+r k(S)$, where $\operatorname{pr}(G)$ denotes the presentation rank of $G$. Thus if $\operatorname{pr}(G)=0$ (e.g. if $G$ is soluble, see [3] 6.9), then $d(G)$ is bounded using the bounds for $r k(S)$ in [10].

Let $N$ denote the soluble radical of $G$. If $\operatorname{pr}(G) \neq 0$, then $d(G / N)=d(G)$ by Theorem B(ii) of [4]. Clearly $H=G / N$ has trivial soluble radical. Then there exists a normal subgroup $E(H)=S_{1} \times S_{2} \times \ldots S_{r}=E$ say of $H$ with $C_{H}(E)=\langle 1\rangle$, where the $S_{i}$ are perfect simple groups and $1 \leq r \leq n / 2$ by Theorem 2 . Now $H$ permutes the $S_{i}$ by conjugation. Applying the arguments from the final two paragraphs of the above proof of Theorem 1, but now to each orbit of $H$ in this action, and using the weak bound $\left[n^{2} / 4\right]$ in Lemma 1 , whose proof does not use [5,8], we can again bound $d(G)$. Specifically we obtain $d(G) \leq 2 t+5 r+\left[r^{2} / 4\right]$, where $t$ is the number of such orbits.

Using $1 \leq t \leq r \leq[n / 2]$ we obtain $d(G) \leq n+\left[n^{2} / 2\right]$ for all $n \geq 3$ except when $n=4=2 r$. If $n=4=2 r$ we only obtain in this way that $d(G) \leq 15<n^{2}$. To obtain the better bound when $n=4=2 r$ we need a more delicate study of the simple groups $S_{1}$ and $S_{2}$. But in this elementary way we have at least bound $\operatorname{rk}(G L(n, p))$ independently of [5,8] by $n^{2}$ always and by $n+\left[n^{2} / 2\right]$ for all $n \neq 4$.

Open Access This article is distributed under the terms of the Creative Commons Attribution 4.0 International License (http://creativecommons.org/licenses/by/4.0/), which permits unrestricted use, distribution, and reproduction in any medium, provided you give appropriate credit to the original author(s) and the source, provide a link to the Creative Commons license, and indicate if changes were made.

\section{References}

1. Aschbacher, M.: Finite Group Theory. Cambridge University Press, Cambridge (1986); re-issued (2008)

2. Curtis, C.W., Reiner, I.: Representation Theory of Finite Groups and Associative Algebras. Wiley, New York (1962)

3. Gruenberg, K.W.: Relation Modules of Finite Groups. Regional Conference Series in Maths, vol. 25. American Mathematical Society, Providence (1976)

4. Gruenberg, K.W.: Groups of non-zero presentation rank. Symp. Math. 17, 215-224 (1976)

5. Guralnick, R.M.: On the number of generators of a finite group. Arch. Math. (Basel) 53, 251-253 (1989)

6. Huppert, B.: Endliche Gruppen I. Springer, Berlin (1967)

7. Kegel, O.H., Wehrfritz, B.A.F.: Locally Finite Groups. North-Holland, Amsterdam (1973)

8. Lucchini, A.: A bound on the number of generators of a finite group. Arch. Math. (Basel) 53, 313-317 (1989)

9. Wehrfritz, B.A.F.: Infinite Linear Groups. Springer, Berlin (1973)

10. Wehrfritz, B.A.F.: The rank of a linear p-group; an apology. J. Lond. Math. Soc. 21, 237-243 (1980)

11. Wehrfritz, B.A.F.: Finite Groups. World Scientific, Singapore (1999)

12. Wehrfritz, B.A.F.: Variants of theorems of Schur, Baer and Hall, Ricerche Mat, to appear 